\title{
Surgical treatment of atrial fibrillation: Predictors of late recurrence
}

\author{
Sydney L. Gaynor, MD \\ Richard B. Schuessler, PhD \\ Marci S. Bailey, RN \\ Yosuke Ishii, MD \\ John P. Boineau, MD \\ Marye J. Gleva, MD \\ James L. Cox, MD \\ Ralph J. Damiano, Jr, MD
}

From the Division of Cardiothoracic Surgery, Washington University School of Medicine, St Louis, Mo.

Supported in part by National Institutes of Health grant no. RO1-HL032257 and grant no. T32-HL007776.

Drs Ralph J. Damiano, Jr, and Richard B. Schuessler serve as consultants with AtriCure Inc.

Read at the Eighty-fourth Annual Meeting of The American Association for Thoracic Surgery, Toronto, Ontario, Canada, April 25-28, 2004.

Received for publication April 23, 2004; revisions received Aug 6, 2004; accepted for publication Aug 18, 2004.

Address for reprints: Ralph J. Damiano, Jr, MD, Suite 3108 Queeny Tower, 1 BarnesJewish Hospital Plaza, St Louis, MO 63110 (E-mail: damianor@msnotes.wustl.edu).

J Thorac Cardiovasc Surg 2005;129:104-11 $0022-5223 / \$ 30.00$

Copyright $\odot 2005$ by The American Association for Thoracic Surgery

doi:10.1016/j.jtcvs.2004.08.042
Objective: The Cox maze procedure was introduced in 1987 for the treatment of atrial fibrillation. This study evaluated the predictors of late atrial fibrillation recurrence in 276 consecutive patients who underwent this procedure at our institution.

Methods: From 1987 through June 2003, 276 patients (79 female and 197 male patients; mean age, $55 \pm 11$ years) underwent the Cox maze procedure. Thirty-three patients had Cox maze procedure I, 16 patients had Cox maze procedure II, and 197 patients had Cox maze procedure III. The last 30 patients underwent a modified procedure (Cox maze procedure IV) with bipolar radiofrequency ablation. There were $113(41 \%)$ patients who had a concomitant operation, most commonly either a mitral valve procedure (19\%) or coronary artery bypass grafting (20\%). Data were analyzed by means of univariate analysis, with preoperative and perioperative variables used as covariates. Patient follow-up was conducted by means of questionnaire, physician examination, and electrocardiographic documentation. All patients had a minimum of 6 months of follow-up.

Results: Patient follow-up was achieved in $92.8 \%$ of cases, with a mean follow-up time of $5.8 \pm 3.6$ years. Risk factors for late atrial fibrillation recurrence were duration of preoperative atrial fibrillation $(P=.01)$ and Cox maze procedure version $(P=.001)$. There was no difference in actuarial 10-year survival between the Cox maze procedure versions.

Conclusion: The Cox maze procedure remains the gold standard for the treatment of atrial fibrillation and has excellent long-term efficacy. The most significant predictor of late recurrence was duration of preoperative atrial fibrillation, suggesting that earlier surgical intervention would further increase efficacy.

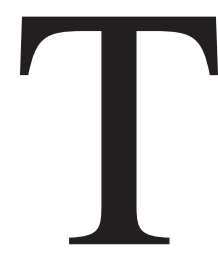

he Cox maze procedure (CMP) was first performed by James L. Cox in 1987. Since then, there have been 4 iterations of this operation. ${ }^{1}$ The long-term success rates of this procedure at our institution have been in excess of $90 \% .^{2}$ The CMP I was effective in eliminating atrial fibrillation (AF) but was discontinued because of the high incidence of chronotropic incompetence and the perceived high rate of pacemaker implantation. The CMP II had similar problems and was also technically difficult because it required transection of the superior vena cava. ${ }^{3}$ Because of the shortcomings of these 2 original versions, the CMP III was introduced and remained the gold standard for the treatment of $\mathrm{AF}$ for more than a decade. ${ }^{4}$ 


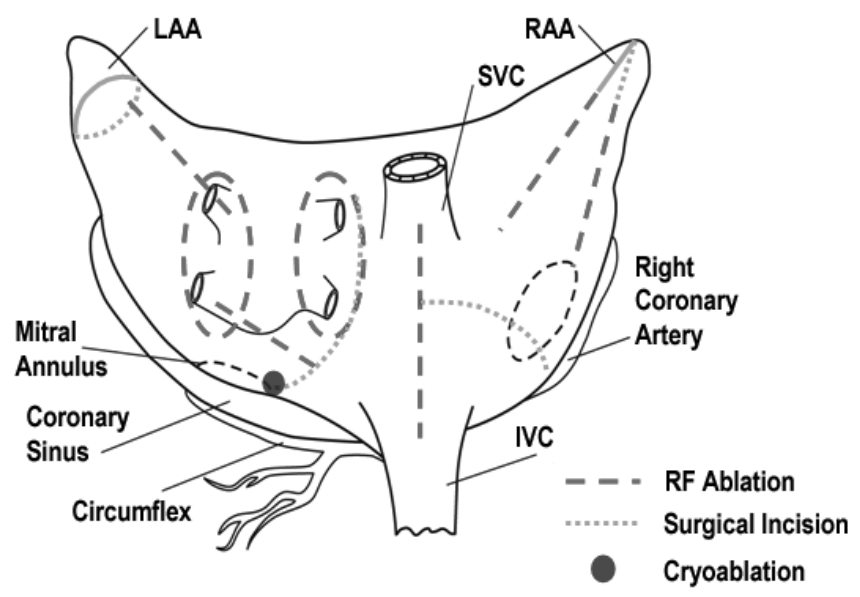

Figure 1. Bipolar radiofrequency-modified CMP IV. LAA, Left atrial appendage; $R A A$, right atrial appendage; $S V C$, superior vena cava; IVC, inferior vena cava; $R F$, radiofrequency.

With the recent introduction of ablation devices, the CMP III was simplified by replacing many of the traditional cut-and-sew lesions with linear lines of ablation by using a bipolar radiofrequency energy device (Atricure, Inc, Cincinnati, Ohio). ${ }^{5-7}$ This new version was first performed in 2002 and has been termed the CMP IV. This procedure differs considerably from the CMP III because it isolates the right and left pulmonary veins as 2 islands (Figure 1). It preserves the right atrial appendage and leaves most of the posterior left atrium in electrical continuity with the remainder of the atrium.

Although the CMP was initially introduced as a lone procedure, it has evolved to the current practice in which it is commonly incorporated with other cardiac procedures, such as valvular and coronary artery bypass surgeries. The efficacy and safety of such concomitant procedures has been reported by our group and others throughout the world. ${ }^{8-11}$ Although the CMP has enjoyed significant clinical success, there have been few reports of factors leading to late recurrence of AF. ${ }^{12,13}$ Despite the fact that our institution has had the world's longest experience with the CMP and its different iterations, we have not previously examined this issue. In this study our prospective database was reviewed to evaluate the predictors of late AF recurrence in 276 consecutive patients who underwent a CMP at Barnes-Jewish Hospital.

\section{Methods and Patients}

From September 1987 through June 2003, 276 patients underwent a CMP. The procedures were performed through a median sternotomy on cardiopulmonary bypass with an arrested heart. Thirtythree patients had a CMP I, 16 patients had a CMP II, 197 patients had a CMP III, and 30 patients had a CMP IV. There were 113 $(41 \%)$ patients who underwent a concomitant cardiac operation, with the most common being either a mitral valve procedure or
TABLE 1. Concomitant cardiac procedures

\begin{tabular}{lcc}
\hline & $\mathbf{n = 1 1 3}$ & \% \\
\hline Mitral valve procedure only & 33 & 29 \\
CABG only & 40 & 35 \\
Mitral valve procedure/CABG & 12 & 10 \\
Aortic valve procedure/CABG & 2 & 2 \\
Tricuspid valve procedure only & 1 & 1 \\
Double-valve procedure & 3 & 3 \\
Double-valve procedure/CABG & 2 & 2 \\
Other procedures* & 20 & 18
\end{tabular}

$C A B G$, Coronary artery bypass grafting.

*Other procedures include atrial septal defect closure, septal myectomy, myxoma resection, and repair of pectus excavatum.

coronary artery bypass grafting (Table 1). The number of patients having concomitant procedures increased over time, with an incidence of $19 \%$ for CMP I, $12 \%$ for the CMP II, $54 \%$ for the CMP III, and $67 \%$ for the CMP IV.

In this series patients presenting with intermittent $\mathrm{AF}$ were defined as having paroxysmal AF. Those presenting with continuous AF were defined as having persistent AF. The patients' clinical profiles and postoperative outcomes were recorded prospectively. More than 400 variables were collected on each patient. The following were considered major perioperative complications: reoperation for bleeding, renal failure requiring dialysis, mediastinitis, placement of an intra-aortic balloon pump, and perioperative transient ischemic attack or stroke. The incidence of early postoperative permanent pacemaker placement (within 30 days of the procedure) was documented. An early postoperative tachyarrhythmia was defined as occurring within the first 30 days and included AF, atrial flutter, and other supraventricular tachycardias.

Patient follow-up was conducted by mailed questionnaire or telephone interview. All patients complaining of AF or those taking warfarin or antiarrhythmic drug therapy had a review of both the referring cardiologist's office charts and a recent electrocardiogram (ECG). A patient was considered to have recurrent AF if they had either self-reported AF episodes or ECG-documented arrhythmias. Late recurrence of $\mathrm{AF}$ was defined as recurrent $\mathrm{AF}-$ atrial flutter occurring at least 6 months after the operation. All deaths occurring outside of the hospital were confirmed by contacting the patient's relatives, the patient's physician, and/or county death records.

Thirteen preoperative and perioperative variables were evaluated in a univariate analysis to identify potential predictors of late AF recurrence. These included age, sex, indication for surgical intervention, type and duration of AF, CMP version, surgical procedure, aortic crossclamp time, cardiopulmonary bypass time, perioperative complications, early atrial tachyarrhythmias, and pacemaker placement. This study was approved by the Washington University School of Medicine Institutional Review Board. Informed consent and permission for release of information were obtained from each participant.

Data were collected and managed in Microsoft Access 2000 and analyzed with SPSS 11.0 software (SPSS, Inc, Chicago, Ill). Normally distributed continuous data were expressed as means \pm SD unless otherwise specified. Nonnormally distributed continu- 


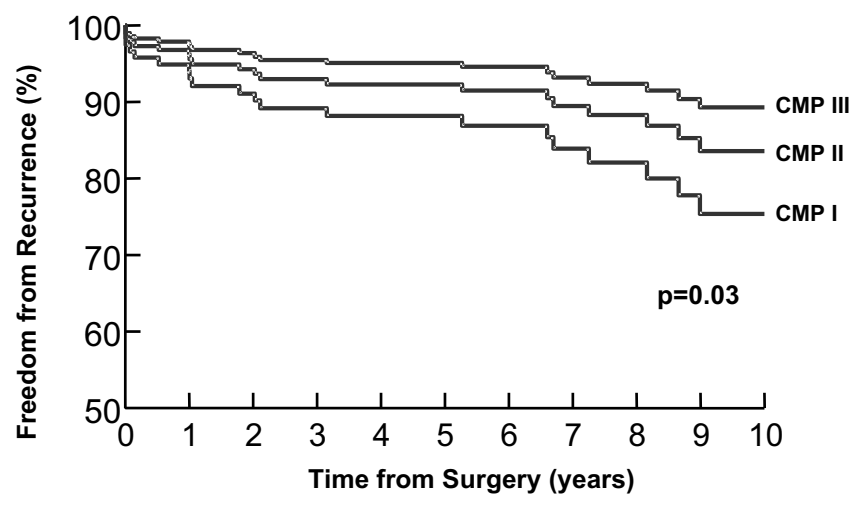

Figure 2. Freedom from late AF recurrence by CMP version.

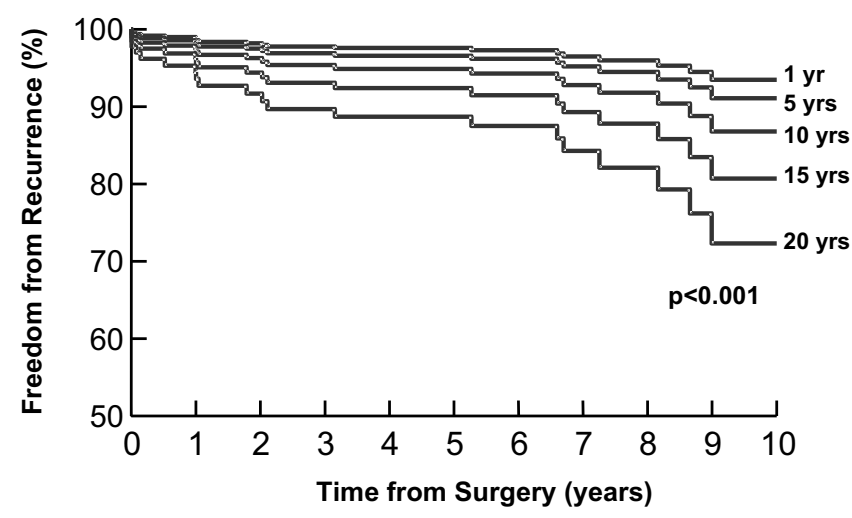

Figure 3. Freedom from late AF recurrence by duration of preoperative AF.

ous data are reported as medians with interquartile ranges (Q25Q75). Univariate analyses were performed for all relevant categoric variables by means of $\chi^{2}$ analysis and $t$ tests or the MannWhitney $U$ test for continuous variables. One-way analysis of variance was used for more than 2 group comparisons. Univariate Cox regression was used to determine which covariates were predictors of late recurrence of AF or survival. Kaplan-Meier estimates were used to depict freedom from $\mathrm{AF}$ and long-term survival. Cox regression analyses were performed by using either duration of preoperative AF or CMP version as covariates to depict their effect on late recurrence of AF (Figures 2 and 3).

\section{Results}

\section{Demographics}

The mean age of our patients undergoing the CMP procedure was $54.9 \pm 11.4$ years (range, 20-80 years; Table 2). There were 197 men and 79 women. Fifty-eight percent of patients were given diagnoses of paroxysmal AF at the time of the operation, with the remainder having persistent AF. The mean duration of AF was $8.2 \pm 7.7$ years, with a range of 2 months to 46 years. The median duration of AF in our series was 5 years. There was no significant difference in the
TABLE 2. Patient characteristics

\begin{tabular}{lcc}
\hline & $\mathbf{n}=\mathbf{2 7 6}$ & $\%$ \\
\hline Age (y) & $54.9 \pm 11.4$ & \\
Male sex & 197 & 71 \\
Paroxysmal AF & 159 & 58 \\
AF duration (y) & & \\
$\quad$ Mean & $8.2 \pm 7.7$ & \\
$\quad$ Median & $5($ IOR, 3-10) & \\
AF cause & & \\
$\quad$ Lone AF & 179 & 65 \\
$\quad$ Valvular disease & 41 & 15 \\
$\quad$ Coronary artery disease & 56 & 20 \\
Indications for surgical intervention & & \\
$\quad$ Arrhythmia intolerance & 196 & 71 \\
$\quad$ Drug failure & 34 & 12 \\
Cerebrovascular event & 46 & 17 \\
\hline
\end{tabular}

$A F$, Atrial fibrillation; IQR, interquartile range.

mean preoperative AF duration by CMP version $(P=.18)$. The indications for surgical intervention were arrhythmia or drug intolerance in $83.3 \%$ (230/276) of patients and cerebrovascular event in $16.7 \%$ (46/276) of patients.

\section{Perioperative Results}

The mean aortic crossclamp time for the entire group was $96 \pm 34$ minutes $(84 \pm 27$ minutes for a lone CMP and 114 \pm 36 minutes for concomitant procedures). The mean cardiopulmonary bypass time for all patients was $183 \pm 44$ minutes (169 \pm 38 minutes for a lone CMP and $203 \pm 45$ minutes for concomitant procedures). The median intensive care unit stay was 2 days. The median hospital length of stay was 11 days. Thirteen percent (37/276) of patients had a major perioperative complication. These complications included reoperation for bleeding in 19 patients, intra-aortic balloon pump placement in 12 patients, renal failure in 5 patients, stroke in 4 patients, and mediastinitis in 4 patients. Six patients experienced more than one major perioperative complication. The 30 -day operative mortality rate was $1.4 \%$ (4/276).

Early postoperative atrial tachyarrhythmias were documented in $44.6 \%$ (123/276) of patients. These postoperative dysrhythmias were usually transient, and $97 \%$ resolved by the 21 st postoperative day. ${ }^{14}$ Early permanent pacemaker placement within 30 days of the operation was required in $16.7 \%(46 / 276)$ of patients. The majority of these patients (78\%) had documented evidence of sick sinus syndrome preoperatively. There was no significant difference in the incidence of postoperative pacemaker implantation between the CMP versions $(P=.16)$.

\section{Late Follow-up}

Late follow-up was achieved in $92.8 \%$ (256/276) of patients, with a mean follow-up duration $6.0 \pm 3.6$ years. The 
TABLE 3. Univariate analysis of patients with late AF recurrence and those free from AF recurrence

\begin{tabular}{|c|c|c|c|}
\hline & $\begin{array}{l}\text { Late } \mathrm{AF} \text { recurrence group } \\
\qquad(\%), \mathrm{n}=20\end{array}$ & $\begin{array}{l}\text { Freedom from late AF group } \\
\qquad(\%), n=236\end{array}$ & $P$ value \\
\hline \multicolumn{4}{|l|}{ Demographics } \\
\hline Age (y) & $59.2 \pm 10.1$ & $55.1 \pm 11.3$ & .11 \\
\hline Male sex & $13(65.0)$ & $169(71.6)$ & .61 \\
\hline Paroxysmal AF & $8(40.0)$ & $139(58.9)$ & .16 \\
\hline AF duration (y) & $16.2 \pm 13.7,10(\operatorname{IOR}, 5-10)$ & $7.6 \pm 6.7,5(\mathrm{IOR}, 3-10)$ & .01 \\
\hline CMP version & & & .001 \\
\hline Preoperative CV event & $7(35.0)$ & $36(15.3)$ & .05 \\
\hline \multicolumn{4}{|l|}{ Perioperative results } \\
\hline Major perioperative complications & $5(25.0)$ & $30(12.7)$ & .17 \\
\hline Hospital LOS (d) & 12 (IOR, 8-21) & $11.0(\operatorname{IQR}, 8-16)$ & .17 \\
\hline Early atrial tachyarrhythmias & $13(65.0)$ & $105(44.5)$ & .10 \\
\hline Early permanent pacemaker & $3(15.0)$ & $41(17.4)$ & 1.0 \\
\hline
\end{tabular}

$A F$, Atrial fibrillation; IQR, interquartile range; CMP, Cox maze procedure; $C V$, cerebrovascular; $L O S$, hospital length of stay.

median follow-up duration was 6.1 years (range, 0.5-15.5 years). The mean follow-up for the CMP I, CMP II, CMP III, and CMP IV groups was 13.7, 9.6, 9.6, and 1.1 years, respectively.

\section{Recurrence of $\mathrm{AF}$}

In our analysis of AF recurrence, all patients identifying themselves as being in AF had confirmation by their cardiologist and an ECG or Holter recording documentation when possible. Late AF recurrence had occurred in $7.8 \%$ (20/256) at last follow-up. Of the patients in sinus rhythm, $19.5 \%$ were taking antiarrhythmic drugs at last follow-up. Six patients required a catheter ablation for either atypical atrial flutter or focal atrial tachycardia during the follow-up period. A univariate analysis identified duration of preoperative $\operatorname{AF}(P=.013)$ and CMP version $(P=.001)$ as the only significant predictors of late AF recurrence (Table 3 ). Patients with a preoperative cerebrovascular event exhibited a trend toward higher recurrence rates of $\mathrm{AF}(P=.054)$.

There was a significant difference in the freedom from AF recurrence over time between CMP versions I, II, and III $(P=.03$, Figure 2$)$. The CMP III had a success rate of $89.3 \%$ at 10 years. In contrast, the CMP I and CMP II were associated with a late freedom from AF recurrence of $75.4 \%$ and $83.6 \%$, respectively. The overall freedom from AF for our entire experience was $92.2 \%$. Patients undergoing the CMP IV had a success rate of $93.1 \%$ at 6 months, but follow-up was too short to allow comparison with previous versions.

Duration of preoperative AF was also a significant predictor. Patients presenting with a preoperative AF duration of 5 years had a $91.1 \%$ freedom from recurrence at 10 years after the CMP. Comparatively, those patients with a preoperative AF duration of 20 years had only a $72.3 \%$ freedom from late $\mathrm{AF}$ recurrence (Figure 3).
There were no other significant predictors of recurrence among the demographic data, including patient age, sex, or type of AF. Perioperative variables, including surgical procedure (lone CMP vs concomitant procedure), aortic crossclamp time, and cardiopulmonary bypass time, were analyzed by using univariate analysis but were not found to be significant predictors of late AF recurrence.

\section{Patient Survival}

The overall survival was $88.7 \%$ at last follow-up. There was no significant difference in actuarial 10-year survival between the various groups. The estimated 10-year survival rates for the CMP I, CMP II, and CMP III groups were $92 \%$, $93 \%$, and $94 \%$, respectively. At last follow-up, patient survival in the CMP IV group was $93.3 \%$.

\section{Discussion}

The CMP has evolved to become the most successful surgical treatment for AF. ${ }^{9,10,15,16}$ Our institution has had the longest experience with the CMP and has maintained a prospective database of our results. Although we have published extensively on our series, this is the first time we have evaluated our database for predictors of late AF recurrence. In this study, the significant predictors of late recurrence of $\mathrm{AF}$ by univariate analysis were duration of preoperative $\mathrm{AF}$ and CMP version.

There has been no previous comparative analysis of late results between the various modifications of the CMP. In this study, there was no significant difference in either operative or late mortality among the 4 different versions of the CMP. There was a larger percentage of patients undergoing concomitant procedures in the CMP III and IV groups when compared with the CMP I and CMP II groups. This is to be expected because the procedure was first applied for the treatment of lone AF. Over the years, the CMP has been 
more frequently incorporated as a concomitant procedure with proven efficacy and low mortality. ${ }^{2,15,16}$ By using a univariate analysis, the CMP I and II groups had a significantly lower success rate than the CMP III group. The CMP IV group had a freedom from AF of $93.3 \%$, but follow-up was too limited to allow a valid comparison with earlier versions.

The reasons for the higher failure rate for the CMP I and II groups are unknown. All 3 procedures had similarly extensive surgical incisions in both atria, and thus it is hard to implicate a specific technical factor. It is possible that there were a greater number of patients in the early versions with untreated sinus node dysfunction who did not receive a permanent pacemaker. These patients might have had a higher incidence of premature atrial contractions, which could serve as a trigger for atrial tachyarrhythmias. ${ }^{17} \mathrm{An}$ other contributing factor might have been a trend $(P=.18)$ toward a longer preoperative $\mathrm{AF}$ duration in the CMP I group (10.6 \pm 9.7 years) compared with the later the CMP III $(8.1 \pm 7.5)$ and CMP IV (7.4 \pm 7.5 years $)$ version.

Preoperative AF duration was also shown to be a significant predictor of late failure. Other investigators have identified duration of preoperative $\mathrm{AF}$ as a predictor of late recurrence after the CMP. ${ }^{18-21}$ Interestingly, patients with AF duration of 5 years had an estimated $91.1 \%$ freedom from $\mathrm{AF}$ at 10 years. This decreased precipitously to $72.3 \%$ for those patients with an AF duration of 20 years. Prolonged AF has been shown to result in electrical remodeling, atrial dilatation, loss of atrial muscle mass, and fibrotic changes occurring within the atrial myocardium. ${ }^{22-25}$ This might be one of the explanations for the higher failure rate. These data would suggest that the earlier referral of patients for CMP would lead to improved surgical results.

A final interesting finding was the trend $(P=.054)$ toward higher recurrence of $\mathrm{AF}$ in patients with a preoperative cerebrovascular event. It is difficult to explain this finding unless there was some associated dysfunction of the autonomic nervous system in these patients. There also might have been confounding covariates that unduly influenced our univariate analysis.

This study had several limitations. One of the major problems was the reliance on the patient's history, referring physician records, and ECGs to determine their rhythm during the follow-up period. Patients who stated that they were in sinus rhythm did not have ECG follow-up. This might have resulted in an underestimation of AF recurrence. However, all of these patients had a previous history of AF, and most were sensitive to their arrhythmia symptomatology. Our follow-up should have captured the majority of treatment failures. It is also possible that patients counted as failures were not actually in AF. It was not possible to obtain ECG confirmation of the arrhythmia in $8(40.0 \%)$ of the 20 failures. Unfortunately, because most of these pa- tients were from outside our region, complete and thorough follow-up was not always possible. Ideally, Holter monitoring of all patients would have been preferred at regular intervals in the postoperative period. However, to our knowledge, these data have never been gathered at any center because of their expense and impracticality.

In our data analysis a univariate model was chosen in preference of a multivariate model because the incidence of AF recurrence was low. This is clearly a limitation; however, it would not be statistically valid to use a multivariate analysis with our relatively small number of treatment failures.

Other investigators have shown that left atrial dimensions (left atrial diameter $\geq 65 \mathrm{~mm}$ ) and $\mathrm{AF}$ wave $(<1.0$ $\mathrm{mm}$ ) have been associated with late AF recurrence. ${ }^{13,20} \mathrm{In}$ this study documentation of left atrial dimension was available in less than one quarter of our patients. This was due to the fact that when the CMP was first introduced, it was not common practice to quantitatively record left atrial dimensions nor was there a standardized method to measure this variable. Similarly, there were no detailed ECG analyses of preoperative atrial activity in these patients. This prevented us from examining their predictive values in this retrospective analysis.

Finally, it was difficult to assess the efficacy of the CMP IV in comparison with the previous CMP versions because the follow-up period was much shorter. Although early results are encouraging, further follow-up will be required to assess long-term efficacy.

In summary, the CMP remains the gold standard for the surgical treatment of AF and has evolved over time into a procedure with excellent long-term efficacy. Preoperative AF duration and CMP version were the only predictors of late recurrence in our database. Our data suggest that an early referral of patients with medically refractory AF for curative surgical therapy would increase the long-term success rates.

\section{References}

1. Cox JL, Schuessler RB, D'Agostino HJ Jr, et al. The surgical treatment of atrial fibrillation, III: development of a definitive surgical procedure. J Thorac Cardiovasc Surg. 1991;101:569-83.

2. Prasad SM, Maniar HS, Camillo CJ, et al. The Cox maze III procedure for atrial fibrillation: long-term efficacy in patients undergoing lone versus concomitant procedures. J Thorac Cardiovasc Surg. 2003;126: 1822-7.

3. Cox JL, Boineau JP, Schuessler RB, Jaquiss RD, Lappas DG. Modification of the maze procedure for atrial flutter and atrial fibrillation. I. Rationale and surgical results. J Thorac Cardiovasc Surg. 1995;110: 473-84.

4. Cox JL. The standard maze-III procedure: In: Cox JL, editor. Operative techniques in thoracic and cardiovascular surgery: a comparative atlas. Vol 5, No 1. Orlando: WB Saunders Co; 2000. p. 2-22.

5. Prasad SM, Maniar HS, Diodato MD, Schuessler RB, Damiano RJ. Physiological consequences of bipolar radiofrequency energy on the atria and pulmonary veins: a chronic animal study. Ann Thorac Surg. 2003;76:836-42. 
6. Gaynor SL, Diodato MD, Prasad SM, et al. A prospective single center clinical trial of a modified Cox maze procedure using bipolar radiofrequency ablation. J Thorac Cardiovasc Surg. 2004;128:535-42.

7. Gillinov AM, McCarthy PM. Atricure bipolar radiofrequency clamp for intraoperative ablation of atrial fibrillation. Ann Thorac Surg. 2002;74:2165-8

8. Sie HT, Beukema WP, Misier AR, et al. Radiofrequency modified maze in patients with atrial fibrillation undergoing concomitant cardiac surgery. J Thorac Cardiovasc Surg. 2001;122:249-56.

9. Schaff HV, Dearani JA, Daly RC, Orszulak TA, Danielson GK. Cox-maze procedure for atrial fibrillation: Mayo Clinic experience. Semin Thorac Cardiovasc Surg. 2000;12:30-7.

10. McCarthy PM, Gillinov AM, Castle L, Chung M, Cosgrove D 3rd. The Cox-maze procedure: the Cleveland Clinic experience. Semin Thorac Cardiovasc Surg. 2000;12:25-9.

11. Nakajima H, Kobayashi J, Bando K, et al. The effect of cryo-maze procedure on early and intermediate term outcome in mitral valve disease: case matched study. Circulation. 2002;106(suppl 1):146-50.

12. Kosakai Y, Kawaguchi AT, Isobe F, et al. Cox maze procedure for chronic atrial fibrillation associated with mitral valve disease. J Thorac Cardiovasc Surg. 1994;108:1049-55.

13. Kamata J, Kawazoe K, Izumoto $\mathrm{H}$, et al. Predictors of sinus rhythm restoration after Cox-maze procedure concomitant with other cardiac operations. Ann Thorac Surg. 1997;64:394-8.

14. Ishii Y, Gleva MJ, Gamache MC, et al. Atrial tachyarrhythmias after the maze procedure. Circulation. 2004;110(Suppl II):II164-8.

15. Millar RC, Arcidi JM Jr, Alison PJM. The Maze III procedure for atrial fibrillation: should the indications be expanded? Ann Thorac Surg. 2000;70:1580-6.

16. Ad N, Cox JL. Combined mitral valve surgery and the Maze III procedure. Semin Thorac Cardiovasc Surg. 2002;14:206-9.

17. Nattel S. New ideas about atrial fibrillation 50 years on. Nature. 2003;415:219-26.

18. Bando K, Kobayashi J, Kosakai Y, et al. Impact of Cox maze procedure on outcome in patients with atrial fibrillation and mitral valve disease. J Thorac Cardiovasc Surg. 2002;124:575-83.

19. Yuda S, Nakatani S, Isobe F, Kosakai Y, Miyatake K. Comparative efficacy of the Maze procedure for restoration of atrial contraction in patients with and without giant left atrium associated with mitral valve disease. J Am Coll Cardiol. 1998;31:1097-102.

20. Kobayashi J, Kosakai Y, Nakano K, Sasako Y, Eishi K, Yamamoto F. Improved success rate of the maze procedure in mitral valve disease by new criteria for patients' selection. Eur J Cardiothorac Surg. 1998; $13: 247-52$

21. Fumitaka I, Yasunaru K. The outcome and indications of the Cox maze III procedure for chronic atrial fibrillation with mitral valve disease. J Thorac Cardiovasc Surg. 1998;116:220-7.

22. Henry WL, Morganroth J, Pearlman AS, et al. Relation between echocardiographically determined left atrial size and atrial fibrillation. Circulation. 1976;53:273-9.

23. Bailey GWH, Braniff BA, Hancock EW, Cohn KE. Relation of left atrial pathology to atrial fibrillation in mitral valvular disease. Ann Intern Med. 1968;69:13-20.

24. Davies MJ, Pomerance A. Pathology of atrial fibrillation in man. $\mathrm{Br}$ Heart J. 1972;34:520-5.

25. Sanfilippo AJ, Abascal VM, Sheehan M, et al. Atrial enlargement as a consequence of atrial fibrillation. A prospective echocardiographic study. Circulation. 1990;82:792-7.

\section{Discussion}

Dr A. Marc Gillinov (Cleveland, Ohio). That was an excellent presentation of very important data from Washington University in St Louis. This is a large experience, nearly 300 patients spanning 16 years. That makes this the longest follow-up of any contemporary surgical or ablative approach to the treatment of AF. The patients might not be entirely representative of those we see in the operating room today, with a preponderance of lone AF and paroxysmal $\mathrm{AF}$ in this series.
In a courageous maneuver that is uncommon, the investigators chose to analyze failures. In fact, to their credit, there were only 20 failures in the entire series, and this precluded a multivariable analysis. Still, though, they came up with 2 very important messages: what we do in the operating room for lesion set matters, and the duration of preoperative AF also matters. These are 2 modifiable factors, factors that we can deal with. I think the data stand for themselves, and I have 3 questions that ask the authors to speculate as to their importance for our future practice.

First, with your experience encompassing 4 different procedures, should different patients with different types of AF have different operations? For example, should paroxysmal AF or continuous AF be treated differently one from the other?

Dr Damiano. Thank you, Dr Gillanov, for your kind comments, and I certainly would like to start by recognizing your important contributions in this area.

I think that is an excellent question, and these data really do not address whether different procedures or possibly more limited procedures might or might not be successful in certain patients. I think the one thing you can take from this series is that with the full lesion set of a CMP III or a CMP IV-and basically all the procedures had very similar lesion sets-that you can get excellent late results. Interestingly, we had no difference in late success, irrespective of whether you had paroxysmal or continuous AF, and this is in distinct contradistinction to the earlier results with catheter-based pulmonary vein isolation.

I think what I would take home from this study is that clearly it is important how long patients are in AF before they are referred to us, and it would certainly suggest that in these patients there is irreversible electrical and anatomic remodeling going on in the atria that, despite this procedure, really prevent long-term success. It is my belief and that of our group that it is very important to communicate this to the cardiologists and that early referral of patients for surgical correction, whether with perhaps a more limited procedure or this procedure, will clearly improve our results. I think if we can get early referral, we might be able to take a more limited approach in certain patients.

Dr Gillinov. My second question concerns the CMP IV. The CMP III has a box lesion around all 4 pulmonary veins, and the CMP IV has 3 sides of the box, and therefore more of the left atrium is in electrical and physical continuity. Is this important?

Dr Damiano. That is an excellent question. Again, I am not sure our data could answer that. I think it is important when you look at this study to remember that the median follow-up in the CMP IV group is only 1.1 years, as opposed to more than 10 years, for instance, in the CMP I group. Thus I think it is a little early to speculate on whether leaving that posterior left atrium in continuity is going to have a positive or a potentially negative late effect on our results, and we are certainly closely continuing to follow that. I would only say that our early results are encouraging that this, which is a quite simpler version of the procedure, has similar short-term efficacy, and we will just have to wait and see whether that is going to hold up in the long term.

Your point is an excellent one, and the one thing we have noticed right away is that the occurrence of earlier atrial tachyarrhythmias is significantly higher in the CMP IV group when compared with the CMP III group, slightly more than $60 \%$ 
compared with approximately $40 \%$ in the CMP III. Therefore this might be due to the fact that we are leaving a lot more atrium in electrical continuity. But interestingly, as this study shows, early atrial tachyarrhythmias, which I think is actually an important point, do not seem to be a significant predictor of late failure.

Dr Gillinov. My final question concerns the right atrial lesions. Most people would say that AF usually comes from the left atrium. Do you believe the right atrial lesions are important for fibrillation or perhaps for flutter?

Dr Damiano. Yes, certainly I think many people are omitting the right atrial lesions, and unfortunately, I would only say that at least in these data, all of the patients in this study had right-sided lesions, and I cannot really comment what the efficacy would be if you left them out. I would say that we have done-and it is a very unrepresentative group but I will give you this anecdotal experience-12 patients in whom we have just done either left-sided lesions or pulmonary vein isolation alone and a left atrial appendectomy, and we saw a postoperative incidence of atrial flutter in that group of approximately $25 \%$. Other experiences around the world-and there are a number of investigators in this room and attending this meeting who have had extensive experience-would indicate perhaps a $10 \%$ to $15 \%$ incidence of late atrial flutter.

Interestingly, we recently have gone back and analyzed some of our mapping data on human AF that was referred. We looked at approximately 21 patients evenly split between paroxysmal and continuous AF, and a third of those patients had their driver focus of the $\mathrm{AF}$ in the right side. Therefore theoretically, if we had not done right-sided lesions, this might have been a group of patients that would have experienced failures.

In my opinion there will be a higher failure rate without the right-sided lesions. I think there are clearly a significant number of patients who have left-sided AF. The problem is we are just not smart enough to identify who those are right now.

Dr Joao Q. Melo (Carnaxide, Portugal). Good article, Ralph. My comment is that we all know that paroxysmal AF and persistent $\mathrm{AF}$ are precursors for permanent $\mathrm{AF}$, and it is the first article in my life in which I can see where paroxysmal AF is not a predictor of a good outcome. And you are also asking and proposing earlier referral, and it is difficult to propose earlier referral if paroxysmal AF is not a predictor of a good outcome. Therefore my question is as follows: can you elaborate on why you found paroxysmal AF not being a predictor of a good outcome, and can that be related with patient selection because that is the only reason I can find for such a finding?

Dr Damiano. Actually, it is an excellent question, and when we look back on our previous study we presented when we just analyzed the CMP III experience, we did not find paroxysmal AF to be a risk factor. Now I think perhaps one difference between our study and others is that all these patients had very extensive lesion sets. With a limited lesion set or a catheter-based approach, I would agree that if you are going to direct most of your attention to the left side, you probably will see differences between paroxysmal and continuous AF. But this is really our results, and we did not see any difference. It does not really come close to being significant, perhaps because we use such an extensive lesion set in our experience at Washington University.
Another thing that is a little unique about our experience compared with that of others is that our average duration of AF is actually quite long because this goes back to span an experience that began when Jim Cox first introduced this operation, and most of those patients had had extremely long-lasting AF. But I do not think that just because we did not show that paroxysmal AF had better results than continuous AF that this should discourage the cardiologists from referring patients earlier.

We have had patients with very long-standing paroxysmal AF in this study, and I think it does show that if you are having AF that you are undergoing probably some type of an irreversible remodeling process and that despite what you do there are some patients who we will not be able to cure. I think it is a message we have to get across to the cardiology community.

Dr Yaron Moshkovitz (Tel-Aviv, Israel). I would like to ask you whether you have got any information regarding the percentage of patients who seem to be in normal sinus rhythm after their operations but in whom the atria still do not contract.

Dr Damiano. That is a very interesting question and continuing to be an important point in the literature when evaluating a surgical series treating AF. The answer to that is we simply do not have data on atrial contractility in this large series.

You noticed we did not present any left atrial size data, which also has been shown to be a strong predictor of failure by Dr Kosakai and others around the world, and we simply did not have adequate left atrial dimensions in all these patients. Our group has previously published that there was impaired atrial contractility in a subgroup of these patients in about $30 \%$ to $40 \%$ of the patients.

I would say we have gone back and looked at that, and the reality is that the great majority of atrial emptying occurs passively, and whether your atria move has very little to add to your atrial ejection fraction. We are looking at this in the laboratory right now with magnetic resonance imaging. As long as you have normal ventricular compliance, more than $80 \%$ of atrial emptying occurs passively with opening of the mitral valve; you do not need an atrial kick. And I would say in this series we only had one late stroke, which would go along with the Cleveland Clinic experience and others. This procedure does eliminate late stroke.

Most of the patients, more than $80 \%$, are off any anticoagulation, and I would argue that whatever their atrial contractility is, it seems to be good enough.

Dr Guo-Wei He (Hong Kong, China). Dr Damiano, I enjoyed your study very much. I work in both the United States and China. Most of the patients with AF we encounter in China are associated with rheumatic heart disease. Therefore I am particularly interested in the cause's effect on the long-term recurrence after the maze procedure with mitral valve disease.

I realize that in your series you have 41 patients with heart valve disease, probably too small a number to further divide into subgroups according to the cause of the valve disease, such as rheumatic or degenerative disease. However, I still would be very interested to know whether there is a trend for those with rheumatic heart disease and severe mitral stenosis associated with $\mathrm{AF}$ for long-standing history. Do they have a higher incidence to have recurrence for $\mathrm{AF}$ or a lower incidence? 
Dr Damiano. That is an excellent question, and you are exactly correct to point out that we do not have enough patients with these problems to probably comment intelligently about that precise risk factor for recurrence. All I can tell you is that in our series the cause of AF was not a risk factor at all for late recurrence. Now you have to remember that until recently we did not have as many patients with rheumatic disease as they have seen in Japan and that obviously you see in China. Therefore we really do not have the data set.

Again, referring back to some beautiful work that has been done in Japan, clearly the large left atrium has a higher rate of recurrence and late failure.

\section{Bound volumes available to subscribers}

Bound volumes of The Journal of Thoracic and Cardiovascular Surgery are available to subscribers (only) for the 2005 issues from the Publisher, at a cost of $\$ 134.00$ for domestic, $\$ 165.85$ for Canadian, and $\$ 155.00$ for international subscribers for Vol 129 (January-June) and Vol 130 (July-December). Shipping charges are included. Each bound volume contains a subject and author index.

The binding is durable buckram with the Journal name, volume number, and year stamped in gold on the spine. Payment must accompany all orders. Contact Elsevier Inc., Subscription Customer Service, 6277 Sea Harbor Dr, Orlando, FL 32887, USA; phone $800-654-2452$ or $407-345-4000$.

Subscriptions must be in force to qualify. Bound volumes are not available in place of a regular Journal subscription. 